\title{
TOPOLOGICAL TRANSVERSALITY PRINCIPLES AND GENERAL COINCIDENCE THEORY
}

\author{
Donal O'Regan
}

\begin{abstract}
This paper presents general topological coincidence principles for multivalued maps defined on subsets of completely regular topological spaces.
\end{abstract}

\section{Introduction.}

The notion of an essential map was introduced by Granas [2] and extended in a variety of setting; see $[1,5,6,7]$ and the references therein. In this paper we present a general continuation theory for coincidences. Our theory relies on a Urysohn type lemma and on the notion of $d-\Phi$-essential and $d-L-\Phi-$ essential maps. In particular we present a general topological transversality type theorem which extends results in the literature; see $[1,3,4,6,7]$ and the references therein.

\section{2. $\quad d-\Phi-$-essential maps.}

Let $E$ be a completely regular topological space and $U$ an open subset of E.

We will consider classes $\mathbf{A}$ and $\mathbf{B}$ of maps.

Key Words: Continuation methods, essential maps, coincidence principles.

2010 Mathematics Subject Classification: Primary 47H10; Secondary 54H25.

Received: February, 2016.

Revised: May, 2016.

Accepted: May, 2016. 
Definition 2.1. We say $F \in A(\bar{U}, E)$ (respectively $F \in B(\bar{U}, E)$ ) if $F: \bar{U} \rightarrow$ $2^{E}$ and $F \in \mathbf{A}(\bar{U}, E)$ (respectively $F \in \mathbf{B}(\bar{U}, E)$ ); here $2^{E}$ denotes the family of nonempty subsets of $E$.

In this section we fix a $\Phi \in B(\bar{U}, E)$.

Definition 2.2. We say $F \in A_{\partial U}(\bar{U}, E)$ if $F \in A(\bar{U}, E)$ with $F(x) \cap \Phi(x)=$ $\emptyset$ for $x \in \partial U$; here $\partial U$ denotes the boundary of $U$ in $E$.

For any map $F \in A(\bar{U}, E)$ let $F^{\star}=I \times F: \bar{U} \rightarrow 2^{\bar{U} \times E}$, with $I: \bar{U} \rightarrow \bar{U}$ given by $I(x)=x$, and let

$$
d:\left\{\left(F^{\star}\right)^{-1}(B)\right\} \cup\{\emptyset\} \rightarrow \Omega
$$

be any map with values in the nonempty set $\Omega$; here $B=\{(x, \Phi(x)): x \in \bar{U}\}$.

Definition 2.3. Let $E$ be a completely regular (respectively normal) topological space, and $U$ an open subset of $E$. Let $F, G \in A_{\partial U}(\bar{U}, E)$. We say $F \cong G$ in $A_{\partial U}(\bar{U}, E)$ if there exists a map $H: \bar{U} \times[0,1] \rightarrow 2^{E}$ with $H(., \eta().) \in A(\bar{U}, E)$ for any continuous function $\eta: \bar{U} \rightarrow[0,1]$ with $\eta(\partial U)=0, H_{t}(x) \cap \Phi(x)=\emptyset$ for any $x \in \partial U$ and $t \in[0,1], H_{1}=F$, $H_{0}=G$ and $\left\{x \in \bar{U}:(x, \Phi(x)) \cap H^{\star}(x, t) \neq \emptyset\right.$ for some $\left.t \in[0,1]\right\}$ is compact (respectively closed); here $H^{\star}(x, t)=(x, H(x, t))$ and $H_{t}(x)=H(x, t)$.

The following conditions will be assumed:

$$
\cong \text { is an equivalence relation in } A_{\partial U}(\bar{U}, E) \text {, }
$$

and

$$
\left\{\begin{array}{l}
\text { if } F, G \in A_{\partial U}(\bar{U}, E) \text { with }\left.F\right|_{\partial U}=\left.G\right|_{\partial U} \text { and } F \cong G \\
\text { in } A_{\partial U}(\bar{U}, E) \text { then } d\left(\left(F^{\star}\right)^{-1}(B)\right)=d\left(\left(G^{\star}\right)^{-1}(B)\right) .
\end{array}\right.
$$

Definition 2.4. Let $F \in A_{\partial U}(\bar{U}, E)$ with $F^{\star}=I \times F$. We say $F^{\star}: \bar{U} \rightarrow$ $2^{\bar{U} \times E}$ is $d-\Phi$-essential if $d\left(\left(F^{\star}\right)^{-1}(B)\right) \neq d(\emptyset)$. We say $F^{\star}$ is $d-\Phi$-inessential if $d\left(\left(F^{\star}\right)^{-1}(B)\right)=d(\emptyset)$.

Remark 2.1. If $F^{\star}$ is $d-\Phi$-essential then

$$
\begin{aligned}
\emptyset \neq\left(F^{\star}\right)^{-1}(B) & =\left\{x \in \bar{U}: F^{\star}(x) \cap B \neq \emptyset\right\} \\
& =\{x \in \bar{U}:(x, F(x)) \cap(x, \Phi(x)) \neq \emptyset\},
\end{aligned}
$$

and this together with $F(x) \cap \Phi(x)=\emptyset$ for $x \in \partial U$ implies that there exists $x \in U$ with $(x, \Phi(x)) \cap F^{\star}(x) \neq \emptyset$ (i.e. $\Phi(x) \cap F(x) \neq \emptyset$ ). 
Theorem 2.1. Let $E$ be a completely regular (respectively normal) topological space, $U$ an open subset of $E, B=\{(x, \Phi(x)): x \in \bar{U}\}, d$ a map defined in (2.1) and assume (2.2) and (2.3) hold. Suppose $F \in A_{\partial U}(\bar{U}, E)$ and assume the following condition holds:

$$
\left\{\begin{array}{l}
\text { if there exists a map } G \in A_{\partial U}(\bar{U}, E) \text { with } G \cong F \text { in } \\
A_{\partial U}(\bar{U}, E) \text { and } d\left(\left(G^{\star}\right)^{-1}(B)\right)=d(\emptyset) \text { with } G^{\star}=I \times G, \\
\text { and if } H \text { is the map defined in Definition } 2.3 \text { and } \\
\mu: \bar{U} \rightarrow[0,1] \text { is any continuous map with } \mu(\partial U)=0, \text { then } \\
\{x \in \bar{U}:(x, \Phi(x)) \cap(x, H(x, t \mu(x))) \neq \emptyset \text { for some } t \in[0,1]\} \\
\text { is closed. }
\end{array}\right.
$$

Then the following are equivalent:

(i). $F^{\star}=I \times F: \bar{U} \rightarrow 2^{\bar{U} \times E}$ is d- $\Phi$-inessential;

(ii). there exists a map $G \in A_{\partial U}(\bar{U}, E)$ with $G^{\star}=I \times G$ and $G \cong F$ in $A_{\partial U}(\bar{U}, E)$ such that $d\left(\left(G^{\star}\right)^{-1}(B)\right)=d(\emptyset)$.

PROOF: (i) implies (ii) is immediate. Next we prove (ii) implies (i). Suppose there exists a map $G \in A_{\partial U}(\bar{U}, E)$ with $G^{\star}=I \times G$ and $G \cong F$ in $A_{\partial U}(\bar{U}, E)$ such that $d\left(\left(G^{\star}\right)^{-1}(B)\right)=d(\emptyset)$. Let $H: \bar{U} \times[0,1] \rightarrow 2^{E}$ with $H(., \eta().) \in A(\bar{U}, E)$ for any continuous function $\eta: \bar{U} \rightarrow[0,1]$ with $\eta(\partial U)=0, H_{t}(x) \cap \Phi(x)=\emptyset$ for any $x \in \partial U$ and $t \in[0,1], H_{1}=G$, $H_{0}=F$ and $\left\{x \in \bar{U}:(x, \Phi(x)) \cap H^{\star}(x, t) \neq \emptyset\right.$ for some $\left.t \in[0,1]\right\}$ is compact (respectively closed); here $H^{\star}(x, t)=(x, H(x, t))$ and $\left.H_{t}(x)=H(x, t)\right)$. Consider

$$
D=\left\{x \in \bar{U}:(x, \Phi(x)) \cap H^{\star}(x, t) \neq \emptyset \text { for some } t \in[0,1]\right\} .
$$

If $D=\emptyset$ then in particular $\emptyset=(x, \Phi(x)) \cap H^{\star}(x, 0)=(x, \Phi(x)) \cap F^{\star}(x)$ for $x \in \bar{U}$ i.e. $\left(F^{\star}\right)^{-1}(B)=\emptyset$ so $d\left(\left(F^{\star}\right)^{-1}(B)\right)=d(\emptyset)$ i.e. $F^{\star}$ is $d-\Phi-$ inessential. Next suppose $D \neq \emptyset$. Note $D$ is compact (respectively closed). Also $D \cap \partial U=\emptyset$ since $H_{t}(x) \cap \Phi(x)=\emptyset$ for any $x \in \partial U$ and $t \in[0,1]$. Thus there exists a continuous map $\mu: \bar{U} \rightarrow[0,1]$ with $\mu(\partial U)=0$ and $\mu(D)=1$. Define a map $R_{\mu}: \bar{U} \rightarrow 2^{E}$ by $R_{\mu}(x)=H(x, \mu(x))=H_{\mu(x)}(x)$ and let $R_{\mu}^{\star}=I \times R_{\mu}$. Note $R_{\mu} \in A(\bar{U}, E),\left.R_{\mu}\right|_{\partial U}=\left.H_{0}\right|_{\partial U}=\left.F\right|_{\partial U}$ since $\mu(\partial U)=0$, and $R_{\mu} \in A_{\partial U}(\bar{U}, E)$.

Also note since $\mu(D)=1$ that

$$
\begin{aligned}
\left(R_{\mu}^{\star}\right)^{-1}(B) & =\{x \in \bar{U}:(x, \Phi(x)) \cap(x, H(x, \mu(x)) \neq \emptyset\} \\
& =\left\{x \in \bar{U}:(x, \Phi(x)) \cap(x, H(x, 1) \neq \emptyset\}=\left(G^{\star}\right)^{-1}\right.
\end{aligned}
$$


so

$$
d\left(\left(R_{\mu}^{\star}\right)^{-1}(B)\right)=d\left(\left(G^{\star}\right)^{-1}(B)\right)=d(\emptyset) .
$$

We claim

$$
R_{\mu} \cong F \text { in } A_{\partial U}(\bar{U}, E) .
$$

If (2.6) is true then (2.3) and (2.5) guarantee that

$$
d\left(\left(F^{\star}\right)^{-1}(B)\right)=d\left(\left(R_{\mu}^{\star}\right)^{-1}(B)\right)=d(\emptyset),
$$

so $F^{\star}$ is $d-\Phi$-inessential.

It remains to show (2.6). Let $Q: \bar{U} \times[0,1] \rightarrow 2^{E}$ be given by $Q(x, t)=$ $H(x, t \mu(x))$. Note $Q(., \eta().) \in A(\bar{U}, E)$ for any continuous function $\eta: \bar{U} \rightarrow$ $[0,1]$ with $\eta(\partial U)=0$ and (see (2.4) and Definition 2.3)

$$
\begin{aligned}
\{x \in \bar{U}: \emptyset & \neq(x, \Phi(x)) \cap(x, Q(x, t)) \\
& =(x, \Phi(x)) \cap(x, H(x, t \mu(x))) \text { for some } t \in[0,1]\}
\end{aligned}
$$

is compact (respectively closed). Note $Q_{0}=F$ and $Q_{1}=R_{\mu}$. Finally if there exists a $t \in[0,1]$ and $x \in \partial U$ with $\Phi(x) \cap Q_{t}(x) \neq \emptyset$ then $\Phi(x) \cap H_{t \mu(x)}(x) \neq \emptyset$, so $x \in D$ and so $\mu(x)=1$ i.e. $\Phi(x) \cap H_{t}(x) \neq \emptyset$, a contradiction. Thus $(2.6)$ holds.

Now Theorem 2.1 immediately yields the following continuation theorem.

Theorem 2.2. Let $E$ be a completely regular (respectively normal) topological space, $U$ an open subset of $E, B=\{(x, \Phi(x)): x \in \bar{U}\}, d$ a map defined in (2.1) and assume (2.2), (2.3) and (2.4) hold. Suppose $J$ and $\Psi$ are two maps in $A_{\partial U}(\bar{U}, E)$ with $J^{\star}=I \times J$ and $\Psi^{\star}=I \times \Psi$ and with $J \cong \Psi$ in $A_{\partial U}(\bar{U}, E)$. Then $J^{\star}$ is $d-\Phi-$ inessential if and only if $\Psi^{\star}$ is $d-\Phi-i n e s s e n t i a l$.

PROOF: Assume $J^{\star}$ is $d-\Phi$-inessential. Then (see Theorem 2.1) there exists a map $Q \in A_{\partial U}(\bar{U}, E)$ with $Q^{\star}=I \times Q$ and $Q \cong J$ in $A_{\partial U}(\bar{U}, E)$ such that $d\left(\left(Q^{\star}\right)^{-1}(B)\right)=d(\emptyset)$. Note (since $\cong$ is an equivalence relation in $\left.A_{\partial U}(\bar{U}, E)\right)$ also that $Q \cong \Psi$ in $A_{\partial U}(\bar{U}, E)$. Then Theorem 2.1 (with $F=\Psi$ and $G=Q$ ) guarantees that $\Psi^{\star}$ is $d-\Phi$-inessential. Similarly if $\Psi^{\star}$ is $d-\Phi-$ inessential then $J^{\star}$ is $d-\Phi$-inessential.

We now show how the ideas in this section can be applied to other natural situations. Let $E$ be a Hausdorff topological vector space (so automatically a completely regular space), $Y$ a topological vector space, and $U$ an open subset of $E$. Also let $L: \operatorname{dom} L \subseteq E \rightarrow Y$ be a linear single valued map; 
here $\operatorname{dom} L$ is a vector subspace of $E$. Finally $T: E \rightarrow Y$ will be a linear single valued map with $L+T: \operatorname{dom} L \rightarrow Y$ a bijection; for convenience we say $T \in H_{L}(E, Y)$.

Definition 2.5. We say $F \in A(\bar{U}, Y ; L, T)$ (respectively $F \in B(\bar{U}, Y ; L, T)$ ) if $F: \bar{U} \rightarrow 2^{Y}$ and $(L+T)^{-1}(F+T) \in A(\bar{U}, E)$ (respectively $(L+T)^{-1}(F+T) \in$ $B(\bar{U}, E))$.

We now fix a $\Phi \in B(\bar{U}, Y ; L, T)$.

Definition 2.6. We say $F \in A_{\partial U}(\bar{U}, Y ; L, T)$ if $F \in A(\bar{U}, Y ; L, T)$ with $(L+T)^{-1}(F+T)(x) \cap(L+T)^{-1}(\Phi+T)(x)=\emptyset$ for $x \in \partial U$.

For any map $F \in A(\bar{U}, Y ; L, T)$ let $F^{\star}=I \times(L+T)^{-1}(F+T): \bar{U} \rightarrow$ $2^{\bar{U} \times E}$, with $I: \bar{U} \rightarrow \bar{U}$ given by $I(x)=x$, and let

$$
d:\left\{\left(F^{\star}\right)^{-1}(B)\right\} \cup\{\emptyset\} \rightarrow \Omega
$$

be any map with values in the nonempty set $\Omega$; here

$$
B=\left\{\left(x,(L+T)^{-1}(\Phi+T)(x)\right): x \in \bar{U}\right\} .
$$

Definition 2.7. Let $F, G \in A_{\partial U}(\bar{U}, Y ; L, T)$. Now $F \cong G$ in $A_{\partial U}(\bar{U}, Y ; L, T)$ if there exists a map $H: \bar{U} \times[0,1] \rightarrow 2^{Y}$ with $(L+T)^{-1}(H(., \eta())+$. $T().) \in A(\bar{U}, E)$ for any continuous function $\eta: \bar{U} \rightarrow[0,1]$ with $\eta(\partial U)=0$, $(L+T)^{-1}\left(H_{t}+T\right)(x) \cap(L+T)^{-1}(\Phi+T)(x)=\emptyset$ for any $x \in \partial U$ and $t \in[0,1]$, $H_{1}=F, H_{0}=G$ and

$$
\left\{x \in \bar{U}:\left(x,(L+T)^{-1}(\Phi+T)(x)\right) \cap H^{\star}(x, t) \neq \emptyset \text { for some } t \in[0,1]\right\}
$$

is compact; here $H_{t}(x)=H(x, t)$ and $H^{\star}(x, \lambda)=\left(x,(L+T)^{-1}(H+T)(x, \lambda)\right)$.

The following conditions will be assumed:

$$
\cong \text { is an equivalence relation in } A_{\partial U}(\bar{U}, Y ; L, T),
$$

and

$$
\left\{\begin{array}{l}
\text { if } F, G \in A_{\partial U}(\bar{U}, Y ; L, T) \text { with }\left.F\right|_{\partial U}=\left.G\right|_{\partial U} \text { and } F \cong G \\
\text { in } A_{\partial U}(\bar{U}, Y ; L, T) \text { then } d\left(\left(F^{\star}\right)^{-1}(B)\right)=d\left(\left(G^{\star}\right)^{-1}(B)\right) .
\end{array}\right.
$$

Definition 2.8. Let $F \in A_{\partial U}(\bar{U}, Y ; L, T)$ with $F^{\star}=I \times(L+T)^{-1}(F+T)$. We say $F^{\star}: \bar{U} \rightarrow 2^{\bar{U} \times E}$ is $d-L-\Phi$-essential if $d\left(\left(F^{\star}\right)^{-1}(B)\right) \neq d(\emptyset)$. We say $F^{\star}$ is $d-L-\Phi$-inessential if $d\left(\left(F^{\star}\right)^{-1}(B)\right)=d(\emptyset)$. 
Theorem 2.3. Let $E$ be a Hausdorff topological vector space, $Y$ a topological vector space, $U$ an open subset of $E, B=\left\{\left(x,(L+T)^{-1}(\Phi+T)(x)\right): x \in \bar{U}\right\}$, $L: \operatorname{dom} L \subseteq E \rightarrow Y$ a linear single valued map, $T \in H_{L}(E, Y)$, d a map defined in (2.7) and assume (2.8) and (2.9) hold. Suppose $F \in A_{\partial U}(\bar{U}, Y ; L, T)$ and assume the following condition holds:

$$
\left\{\begin{array}{l}
\text { if there exists a map } G \in A_{\partial U}(\bar{U}, Y ; L, T) \text { with } G \cong F \\
\text { in } A_{\partial U}(\bar{U}, Y ; L, T) \text { and } d\left(\left(G^{\star}\right)^{-1}(B)\right)=d(\emptyset) \text { with } \\
G^{\star}=I \times(L+T)^{-1}(G+T) \text { and if } H \text { is the map } \\
\text { defined in Definition } 2.7 \text { and } \mu: \bar{U} \rightarrow[0,1] \text { is any } \\
\text { continuous map with } \mu(\partial U)=0, \text { then } \\
\left\{x \in \bar{U}: \emptyset \neq\left(x,(L+T)^{-1}(\Phi+T)(x)\right)\right. \\
\left.\cap\left(x,(L+T)^{-1}\left(H_{t \mu(x)}+T\right)(x)\right) \text { for some } t \in[0,1]\right\} \\
\text { is closed. }
\end{array}\right.
$$

Then the following are equivalent:

(i). $F^{\star}=I \times(L+T)^{-1}(F+T): \bar{U} \rightarrow 2^{\bar{U} \times E}$ is $d-L-\Phi-$ inessential;

(ii). there exists a map $G \in A_{\partial U}(\bar{U}, Y ; L, T)$ with $G^{\star}=I \times(L+T)^{-1}(G+T)$ and $G \cong F$ in $A_{\partial U}(\bar{U}, Y ; L, T)$ such that $d\left(\left(G^{\star}\right)^{-1}(B)\right)=d(\emptyset)$.

PROOF: (i) implies (ii) is immediate. Next we prove (ii) implies (i). Suppose there exists a map $G \in A_{\partial U}(\bar{U}, Y ; L, T)$ with $G^{\star}=I \times(L+T)^{-1}(G+T)$ and $G \cong F$ in $A_{\partial U}(\bar{U}, Y ; L, T)$ such that $d\left(\left(G^{\star}\right)^{-1}(B)\right)=d(\emptyset)$. Let $H$ : $\bar{U} \times[0,1] \rightarrow 2^{Y}$ be a map with $(L+T)^{-1}(H(., \eta())+.T().) \in A(\bar{U}, E)$ for any continuous function $\eta: \bar{U} \rightarrow[0,1]$ with $\eta(\partial U)=0,(L+T)^{-1}\left(H_{t}+\right.$ $T)(x) \cap(L+T)^{-1}(\Phi+T)(x)=\emptyset$ for any $x \in \partial U$ and $t \in[0,1], H_{1}=G$, $H_{0}=F\left(\right.$ here $\left.H_{t}(x)=H(x, t)\right)$ and

$$
\left\{x \in \bar{U}:\left(x,(L+T)^{-1}(\Phi+T)(x)\right) \cap H^{\star}(x, t) \neq \emptyset \text { for some } t \in[0,1]\right\}
$$

is compact; here $H^{\star}(x, \lambda)=\left(x,(L+T)^{-1}(H+T)(x, \lambda)\right)$.

Let

$D=\left\{x \in \bar{U}:\left(x,(L+T)^{-1}(\Phi+T)(x)\right) \cap H^{\star}(x, t) \neq \emptyset\right.$ for some $\left.t \in[0,1]\right\}$.

If $D=\emptyset$ then in particular $\left(H_{0}=F\right)$ note $\emptyset=\left(x,(L+T)^{-1}(\Phi+T)(x)\right) \cap$ $\left(x,(L+T)^{-1}(F+T)(x)\right)$, so $F^{\star}$ in $d-L-\Phi$-inessential. Next suppose $D \neq \emptyset$. Note $D$ is compact and $D \cap \partial U=\emptyset$, so there exists a continuous map $\mu$ : $\bar{U} \rightarrow[0,1]$ with $\mu(\partial U)=0$ and $\mu(D)=1$. Define a map $R_{\mu}: \bar{U} \rightarrow 2^{Y}$ by $R_{\mu}(x)=H(x, \mu(x))=H_{\mu(x)}(x)$ and let $R_{\mu}^{\star}=I \times(L+T)^{-1}\left(R_{\mu}+\right.$ $T)$. Notice $R_{\mu} \in A(\bar{U}, Y ; L, T),\left.\quad R_{\mu}\right|_{\partial U}=\left.H_{0}\right|_{\partial U}=\left.F\right|_{\partial U}$ since $\mu(\partial U)=$ 
0 , and $R_{\mu} \in A_{\partial U}(\bar{U}, Y ; L, T)$. Also since $\mu(D)=1$ it is easy to see that $\left(R_{\mu}^{\star}\right)^{-1}(B)=\left(G^{\star}\right)^{-1}(B)$, so $d\left(\left(R_{\mu}^{\star}\right)^{-1}(B)\right)=d\left(\left(G^{\star}\right)^{-1}(B)\right)=d(\emptyset)$. Also note $R_{\mu} \cong F$ in $A_{\partial U}(\bar{U}, Y ; L, T)$ (to see this let $Q: \bar{U} \times[0,1] \rightarrow 2^{Y}$ be given by $Q(x, t)=H(x, t \mu(x)))$, so $d\left(\left(F^{\star}\right)^{-1}(B)\right)=d\left(\left(R_{\mu}^{\star}\right)^{-1}(B)\right)=$ $d(\emptyset)$, and so $F^{\star}$ in $d-L-\Phi$-inessential.

We have immediately the following result.

Theorem 2.4. Let $E$ be a Hausdorff topological vector space, $Y$ a topological vector space, $U$ an open subset of $E, B=\left\{\left(x,(L+T)^{-1}(\Phi+T)(x)\right): x \in\right.$ $\bar{U}\}, L: \operatorname{dom} L \subseteq E \rightarrow Y$ a linear single valued map, $T \in H_{L}(E, Y), d a$ map defined in (2.7) and assume (2.8), (2.9) and (2.10) hold. Suppose $J$ and $\Psi$ are two maps in $A_{\partial U}(\bar{U}, Y ; L, T)$ with $J^{\star}=I \times(L+T)^{-1}(J+T)$ and $\Psi^{\star}=I \times(L+T)^{-1}(\Psi+T)$ and with $J \cong \Psi$ in $A_{\partial U}(\bar{U}, Y ; L, T)$. Then $J^{\star}$ is $d-L-\Phi-$ inessential if and only if $\Psi^{\star}$ is $d-L-\Phi-$ inessential.

Remark 2.2. If $E$ is a normal topological vector space then the assumption that

$$
\left\{x \in \bar{U}:\left(x,(L+T)^{-1}(\Phi+T)(x)\right) \cap H^{\star}(x, t) \neq \emptyset \text { for some } t \in[0,1]\right\}
$$

is compact, can be replaced by

$$
\left\{x \in \bar{U}:\left(x,(L+T)^{-1}(\Phi+T)(x)\right) \cap H^{\star}(x, t) \neq \emptyset \text { for some } t \in[0,1]\right\}
$$

is closed, in Definition 2.7.

Next we discuss the situation when (2.3) is not assumed. To obtain an analogue of Theorem 2.1 and Theorem 2.2 we change the definition of $d-\Phi-$ essential in Definition 2.4.

Definition 2.9. Let $F \in A_{\partial U}(\bar{U}, E)$ with $F^{\star}=I \times F$. We say $F^{\star}: \bar{U} \rightarrow$ $2^{\bar{U} \times E}$ is $d-\Phi$-essential if for every map $J \in A_{\partial U}(\bar{U}, E)$ with $J^{\star}=I \times J$ and $\left.J\right|_{\partial U}=\left.F\right|_{\partial U}$ and $J \cong F$ in $A_{\partial U}(\bar{U}, E)$ we have that $d\left(\left(F^{\star}\right)^{-1}(B)\right)=$ $d\left(\left(J^{\star}\right)^{-1}(B)\right) \neq d(\emptyset)$. Otherwise $F^{\star}$ is $d-\Phi$-inessential. It is immediate that this means either $d\left(\left(F^{\star}\right)^{-1}(B)\right)=d(\emptyset)$ or there exists a map $J \in A_{\partial U}(\bar{U}, E)$ with $J^{\star}=I \times J$ and $\left.J\right|_{\partial U}=\left.F\right|_{\partial U}$ and $J \cong F$ in $A_{\partial U}(\bar{U}, E)$ such that $d\left(\left(F^{\star}\right)^{-1}(B)\right) \neq d\left(\left(J^{\star}\right)^{-1}(B)\right)$.

Theorem 2.5. Let $E$ be a completely regular (respectively normal) topological space, $U$ an open subset of $E, B=\{(x, \Phi(x)): x \in \bar{U}\}, d$ a map defined 
in (2.1) and assume (2.2) holds. Suppose $F \in A_{\partial U}(\bar{U}, E)$ and assume the following condition holds:

$$
\begin{aligned}
& \text { if there exists a map } G \in A_{\partial U}(\bar{U}, E) \text { with } G \cong F \text { in } \\
& A_{\partial U}(\bar{U}, E) \text { and } d\left(\left(F^{\star}\right)^{-1}(B)\right) \neq d\left(\left(G^{\star}\right)^{-1}(B)\right) \\
& \text { with } G^{\star}=I \times G, F^{\star}=I \times F, \text { and if } H \text { is the map } \\
& \text { defined in Definition } 2.3 \text { and } \mu: \bar{U} \rightarrow[0,1] \text { is any } \\
& \text { continuous map with } \mu(\partial U)=0 \text {, then } \\
& \{x \in \bar{U}:(x, \Phi(x)) \cap(x, H(x, t \mu(x))) \neq \emptyset \text { for some } t \in[0,1]\}
\end{aligned}
$$

Then the following are equivalent:

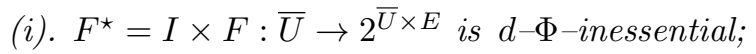

(ii). $d\left(\left(F^{\star}\right)^{-1}(B)\right)=d(\emptyset)$ or there exists a map $G \in A_{\partial U}(\bar{U}, E)$ with $G^{\star}=$ $I \times G$ and $G \cong F$ in $A_{\partial U}(\bar{U}, E)$ such that $d\left(\left(F^{\star}\right)^{-1}(B)\right) \neq d\left(\left(G^{\star}\right)^{-1}(B)\right)$.

PROOF: (i) implies (ii) is immediate. Next we prove (ii) implies (i). If $d\left(\left(F^{\star}\right)^{-1}(B)\right)=d(\emptyset)$ then trivially (i) is true. Next suppose there exists a map $G \in A_{\partial U}(\bar{U}, E)$ with $G^{\star}=I \times G$ and $G \cong F$ in $A_{\partial U}(\bar{U}, E)$ such that $d\left(\left(F^{\star}\right)^{-1}(B)\right) \neq d\left(\left(G^{\star}\right)^{-1}(B)\right)$. Let $H: \bar{U} \times[0,1] \rightarrow 2^{E}$ with $H(., \eta().) \in A(\bar{U}, E)$ for any continuous function $\eta: \bar{U} \rightarrow[0,1]$ with $\eta(\partial U)=0, H_{t}(x) \cap \Phi(x)=\emptyset$ for any $x \in \partial U$ and $t \in[0,1], H_{1}=G, H_{0}=F$ and

$$
\left\{x \in \bar{U}:(x, \Phi(x)) \cap H^{\star}(x, t) \neq \emptyset \text { for some } t \in[0,1]\right\}
$$

is compact (respectively closed); here $H^{\star}(x, t)=(x, H(x, t))$ and $H_{t}(x)=$ $H(x, t))$. Consider

$$
D=\left\{x \in \bar{U}:(x, \Phi(x)) \cap H^{\star}(x, t) \neq \emptyset \text { for some } t \in[0,1]\right\} .
$$

If $D=\emptyset$ then as in Theorem 2.1 we obtain immediately that $d\left(\left(F^{\star}\right)^{-1}(B)\right)=$ $d(\emptyset)$ i.e. $F^{\star}$ is $d-\Phi$-inessential. Next suppose $D \neq \emptyset$. Note $D$ is compact (respectively closed). Also $D \cap \partial U=\emptyset$ and there exists a continuous map $\mu: \bar{U} \rightarrow[0,1]$ with $\mu(\partial U)=0$ and $\mu(D)=1$. Define a map $R_{\mu}: \bar{U} \rightarrow 2^{E}$ by $R_{\mu}(x)=H(x, \mu(x))=H_{\mu(x)}(x)$ and let $R_{\mu}^{\star}=I \times R_{\mu}$. Note $R_{\mu} \in A(\bar{U}, E),\left.R_{\mu}\right|_{\partial U}=\left.H_{0}\right|_{\partial U}=\left.F\right|_{\partial U}$ since $\mu(\partial U)=0$, and $R_{\mu} \in$ $A_{\partial U}(\bar{U}, E)$. Also since $\mu(D)=1$ we have (see Theorem 2.1) $\left(R_{\mu}^{\star}\right)^{-1}(B)=$ $\left(G^{\star}\right)^{-1}(B)$, so $d\left(\left(R_{\mu}^{\star}\right)^{-1}(B)\right)=d\left(\left(G^{\star}\right)^{-1}(B)\right)$. Thus $d\left(\left(F^{\star}\right)^{-1}(B)\right) \neq$ 
$d\left(\left(R_{\mu}^{\star}\right)^{-1}(B)\right)$. Also note (as in Theorem 2.1) $R_{\mu} \cong F$ in $A_{\partial U}(\bar{U}, E)$ (to see this let $Q: \bar{U} \times[0,1] \rightarrow 2^{E}$ be given by $\left.Q(x, t)=H(x, t \mu(x))\right)$. Consequently $F^{\star}$ is $d-\Phi$-inessential (take $J=R_{\mu}$ in Definition 2.9).

Theorem 2.6. Let $E$ be a completely regular (respectively normal) topological space, $U$ an open subset of $E, B=\{(x, \Phi(x)): x \in \bar{U}\}, d$ a map defined in (2.1) and assume (2.2) and (2.11) hold. Suppose $R$ and $\Psi$ are two maps in $A_{\partial U}(\bar{U}, E)$ with $R^{\star}=I \times R$ and $\Psi^{\star}=I \times \Psi$ and with $R \cong \Psi$ in $A_{\partial U}(\bar{U}, E)$. Then $R^{\star}$ is $d-\Phi-$ inessential if and only if $\Psi^{\star}$ is $d-\Phi$-inessential.

PROOF: Assume $R^{\star}$ is $d-\Phi$-inessential.

Then (see Theorem 2.5) either $d\left(\left(R^{\star}\right)^{-1}(B)\right)=d(\emptyset)$ or there exists a map $Q \in A_{\partial U}(\bar{U}, E)$ with $Q^{\star}=I \times Q$ and $Q \cong R$ in $A_{\partial U}(\bar{U}, E)$ such that $d\left(\left(R^{\star}\right)^{-1}(B)\right) \neq d\left(\left(Q^{\star}\right)^{-1}(B)\right)$.

Suppose first that $d\left(\left(R^{\star}\right)^{-1}(B)\right)=d(\emptyset)$. There are two cases to consider, either $d\left(\left(\Psi^{\star}\right)^{-1}(B)\right) \neq d(\emptyset)$ or $d\left(\left(\Psi^{\star}\right)^{-1}(B)\right)=d(\emptyset)$.

Case (1). Suppose $d\left(\left(\Psi^{\star}\right)^{-1}(B)\right) \neq d(\emptyset)$.

Then $d\left(\left(R^{\star}\right)^{-1}(B)\right) \neq d\left(\left(\Psi^{\star}\right)^{-1}(B)\right)$ and we know $R \cong \Psi$ in $A_{\partial U}(\bar{U}, E)$. Now Theorem 2.5 (with $F=\Psi$ and $G=R$ ) guarantees that $\Psi^{\star}$ is $d-\Phi$ inessential.

Case (2). Suppose $d\left(\left(\Psi^{\star}\right)^{-1}(B)\right)=d(\emptyset)$.

Then by definition $\Psi^{\star}$ is $d-\Phi$-inessential.

Next suppose there exists a map $Q \in A_{\partial U}(\bar{U}, E)$ with $Q^{\star}=I \times Q$ and $Q \cong R$ in $A_{\partial U}(\bar{U}, E)$ such that $d\left(\left(R^{\star}\right)^{-1}(B)\right) \neq d\left(\left(Q^{\star}\right)^{-1}(B)\right)$. Note (since $\cong$ is an equivalence relation in $A_{\partial U}(\bar{U}, E)$ ) also that $Q \cong \Psi$ in $A_{\partial U}(\bar{U}, E)$. There are two cases to consider, either $d\left(\left(Q^{\star}\right)^{-1}(B)\right) \neq$ $d\left(\left(\Psi^{\star}\right)^{-1}(B)\right)$ or $d\left(\left(Q^{\star}\right)^{-1}(B)\right)=d\left(\left(\Psi^{\star}\right)^{-1}(B)\right)$.

Case (1). Suppose $d\left(\left(Q^{\star}\right)^{-1}(B)\right) \neq d\left(\left(\Psi^{\star}\right)^{-1}(B)\right)$.

Then Theorem 2.5 (with $F=\Psi$ and $G=Q$ ) guarantees that $\Psi^{\star}$ is $d-\Phi-$ inessential.

Case (2). Suppose $d\left(\left(Q^{\star}\right)^{-1}(B)\right)=d\left(\left(\Psi^{\star}\right)^{-1}(B)\right)$.

Then $d\left(\left(R^{\star}\right)^{-1}(B)\right) \neq d\left(\left(\Psi^{\star}\right)^{-1}(B)\right)$ and we know $R \cong \Psi$ in $A_{\partial U}(\bar{U}, E)$. Now Theorem 2.5 (with $F=\Psi$ and $G=R$ ) guarantees that $\Psi^{\star}$ is $d-\Phi$ - 
inessential.

Thus in all cases $\Psi^{\star}$ is $d-\Phi$-inessential.

Similarly if $\Psi^{\star}$ is $d-\Phi$-inessential then $R^{\star}$ is $d-\Phi$-inessential.

Next we discuss the situation when (2.9) is not assumed. To obtain an analogue of Theorem 2.3 and Theorem 2.4 we change the definition of $d-L$ $\Phi$-essential in Definition 2.8.

Definition 2.10. Let $F \in A_{\partial U}(\bar{U}, Y ; L, T)$ with $F^{\star}=I \times(L+T)^{-1}(F+$ $T)$. We say $F^{\star}: \bar{U} \rightarrow 2^{\bar{U} \times E}$ is $d-L-\Phi$-essential if for every map $J \in$ $A_{\partial U}(\bar{U}, Y ; L, T)$ with $J^{\star}=I \times(L+T)^{-1}(J+T)$ and $\left.J\right|_{\partial U}=\left.F\right|_{\partial U}$ and $J \cong F$ in $A_{\partial U}(\bar{U}, Y ; L, T)$ we have that $d\left(\left(F^{\star}\right)^{-1}(B)\right)=d\left(\left(J^{\star}\right)^{-1}(B)\right) \neq$ $d(\emptyset)$. Otherwise $F^{\star}$ is $d-L-\Phi$-inessential. It is immediate that this means either $d\left(\left(F^{\star}\right)^{-1}(B)\right)=d(\emptyset)$ or there exists a map $J \in A_{\partial U}(\bar{U}, Y ; L, T)$ with $J^{\star}=I \times(L+T)^{-1}(J+T)$ and $\left.J\right|_{\partial U}=\left.F\right|_{\partial U}$ and $J \cong F$ in $A_{\partial U}(\bar{U}, Y ; L, T)$ such that $d\left(\left(F^{\star}\right)^{-1}(B)\right) \neq d\left(\left(J^{\star}\right)^{-1}(B)\right)$.

Theorem 2.7. Let $E$ be a Hausdorff topological vector space, $Y$ a topological vector space, $U$ an open subset of $E, B=\left\{\left(x,(L+T)^{-1}(\Phi+T)(x)\right): x \in \bar{U}\right\}$, $L: \operatorname{dom} L \subseteq E \rightarrow Y$ a linear single valued map, $T \in H_{L}(E, Y)$, d a map defined in (2.7) and assume (2.8) holds. Suppose $F \in A_{\partial U}(\bar{U}, Y ; L, T)$ and assume the following condition holds:

$$
\left\{\begin{array}{l}
\text { if there exists a map } G \in A_{\partial U}(\bar{U}, Y ; L, T) \text { with } G \cong F \text { in } \\
A_{\partial U}(\bar{U}, Y ; L, T) \text { and } d\left(\left(G^{\star}\right)^{-1}(B)\right) \neq d\left(\left(G^{\star}\right)^{-1}(B)\right) \text { with } \\
G^{\star}=I \times(L+T)^{-1}(G+T), F^{\star}=I \times(L+T)^{-1}(F+T), \\
\text { and if } H \text { is the map defined in Definition } 2.7 \text { and } \\
\mu: \bar{U} \rightarrow[0,1] \text { is any continuous map with } \mu(\partial U)=0, \text { then } \\
\left\{x \in \bar{U}: \emptyset \neq\left(x,(L+T)^{-1}(\Phi+T)(x)\right)\right. \\
\left.\cap\left(x,(L+T)^{-1}\left(H_{t \mu(x)}+T\right)(x)\right) \text { for some } t \in[0,1]\right\} \\
\text { is closed. }
\end{array}\right.
$$

Then the following are equivalent:

(i). $F^{\star}=I \times(L+T)^{-1}(F+T): \bar{U} \rightarrow 2^{\bar{U} \times E}$ is $d-L-\Phi-$ inessential;

(ii). $d\left(\left(F^{\star}\right)^{-1}(B)\right)=d(\emptyset)$ or there exists a map $G \in A_{\partial U}(\bar{U}, Y ; L, T)$ with $G^{\star}=I \times(L+T)^{-1}(G+T)$ and $G \cong F$ in $A_{\partial U}(\bar{U}, Y ; L, T)$ such that $d\left(\left(F^{\star}\right)^{-1}(B)\right) \neq d\left(\left(G^{\star}\right)^{-1}(B)\right)$.

PROOF: (i) implies (ii) is immediate. Next we prove (ii) implies (i). If 
$d\left(\left(F^{\star}\right)^{-1}(B)\right)=d(\emptyset)$ then trivially (i) is true. Next suppose there exists a map $G \in A_{\partial U}(\bar{U}, Y ; L, T)$ with $G^{\star}=I \times(L+T)^{-1}(G+T)$ and $G \cong F$ in $A_{\partial U}(\bar{U}, Y ; L, T)$ such that $d\left(\left(F^{\star}\right)^{-1}(B)\right) \neq d\left(\left(G^{\star}\right)^{-1}(B)\right)$. Let $H: \bar{U} \times[0,1] \rightarrow 2^{Y}$ be a map with $(L+T)^{-1}(H(., \eta())+.T().) \in A(\bar{U}, E)$ for any continuous function $\eta: \bar{U} \rightarrow[0,1]$ with $\eta(\partial U)=0,(L+T)^{-1}\left(H_{t}+\right.$ $T)(x) \cap(L+T)^{-1}(\Phi+T)(x)=\emptyset$ for any $x \in \partial U$ and $t \in[0,1], H_{1}=G$, $H_{0}=F\left(\right.$ here $\left.H_{t}(x)=H(x, t)\right)$ and

$$
\left\{x \in \bar{U}:\left(x,(L+T)^{-1}(\Phi+T)(x)\right) \cap H^{\star}(x, t) \neq \emptyset \text { for some } t \in[0,1]\right\}
$$

is compact; here $H^{\star}(x, \lambda)=\left(x,(L+T)^{-1}(H+T)(x, \lambda)\right)$.

Let

$D=\left\{x \in \bar{U}:\left(x,(L+T)^{-1}(\Phi+T)(x)\right) \cap H^{\star}(x, t) \neq \emptyset\right.$ for some $\left.t \in[0,1]\right\}$.

If $D=\emptyset$ then as in Theorem 2.3 we have $d\left(\left(F^{\star}\right)^{-1}(B)\right)=d(\emptyset)$ so $F^{\star}$ in $d-$ $L-\Phi$-inessential. Next suppose $D \neq \emptyset$. Note $D$ is compact and $D \cap \partial U=\emptyset$, so there exists a continuous map $\mu: \bar{U} \rightarrow[0,1]$ with $\mu(\partial U)=0$ and $\mu(D)=1$. Define a map $R_{\mu}: \bar{U} \rightarrow 2^{Y}$ by $R_{\mu}(x)=H(x, \mu(x))=H_{\mu(x)}(x)$ and let $R_{\mu}^{\star}=I \times(L+T)^{-1}\left(R_{\mu}+T\right)$. Notice $R_{\mu} \in A(\bar{U}, Y ; L, T),\left.R_{\mu}\right|_{\partial U}=\left.H_{0}\right|_{\partial U}=$ $\left.F\right|_{\partial U}$ since $\mu(\partial U)=0$, and $R_{\mu} \in A_{\partial U}(\bar{U}, Y ; L, T)$. Also since $\mu(D)=1$ we have $\left(R_{\mu}^{\star}\right)^{-1}(B)=\left(G^{\star}\right)^{-1}(B)$, so $d\left(\left(R_{\mu}^{\star}\right)^{-1}(B)\right)=d\left(\left(G^{\star}\right)^{-1}(B)\right)$. Thus $d\left(\left(F^{\star}\right)^{-1}(B)\right) \neq d\left(\left(R_{\mu}^{\star}\right)^{-1}(B)\right)$. Also note $R_{\mu} \cong F$ in $A_{\partial U}(\bar{U}, Y ; L, T)$ (to see this let $Q: \bar{U} \times[0,1] \rightarrow 2^{Y}$ be given by $Q(x, t)=H(x, t \mu(x))$ ). Consequently $F^{\star}$ is $d-L-\Phi$-inessential.

Theorem 2.8. Let $E$ be a Hausdorff topological vector space, $Y$ a topological vector space, $U$ an open subset of $E, B=\left\{\left(x,(L+T)^{-1}(\Phi+T)(x)\right): x \in\right.$ $\bar{U}\}, L: \operatorname{dom} L \subseteq E \rightarrow Y$ a linear single valued map, $T \in H_{L}(E, Y)$, d a map defined in (2.7) and assume (2.8), and (2.12) hold. Suppose $R$ and $\Psi$ are two maps in $A_{\partial U}(\bar{U}, Y ; L, T)$ with $R^{\star}=I \times(L+T)^{-1}(R+T)$ and $\Psi^{\star}=I \times(L+T)^{-1}(\Psi+T)$ and with $R \cong \Psi$ in $A_{\partial U}(\bar{U}, Y ; L, T)$. Then $R^{\star}$ is $d-L-\Phi-$ inessential if and only if $\Psi^{\star}$ is $d-L-\Phi-$ inessential.

Remark 2.3. If $E$ is a normal topological vector space then the assumption that

$$
\left\{x \in \bar{U}:\left(x,(L+T)^{-1}(\Phi+T)(x)\right) \cap H^{\star}(x, t) \neq \emptyset \text { for some } t \in[0,1]\right\}
$$

is compact, can be replaced by

$$
\left\{x \in \bar{U}:\left(x,(L+T)^{-1}(\Phi+T)(x)\right) \cap H^{\star}(x, t) \neq \emptyset \text { for some } t \in[0,1]\right\}
$$


is closed, in Definition 2.7.

\section{References}

[1]. L. Gorniewicz, Topological fixed point theory of multivalued mappings, Kluwer Acad. Publishers, Dordrecht, 1999.

[2]. A. Granas and J. Dugundji, Fixed Point Theory, Springer-Verlag, New York, 2003.

[3]. D. O'Regan, A unified theory for homotopy principles for multimaps, Applicable Analysis, 92(2013), 1944-1958.

[4]. D. O'Regan, Continuation principles based on essential maps and topological degree, Applicable Analysis, 94(2015), 1032-1041.

[5]. D. O'Regan, Generalized coincidence theory for set-valued maps, Journal of Nonlinear Science and Applications, 10(2017), 855-864.

[6]. D.O'Regan and R. Precup, Theorems of Leray-Schauder Type and Applications, Taylor and Francis Publishers, London, 2002.

[7]. R. Precup, On the topological transversality principle, Nonlinear Anal., 20(1993), 1-9.

Donal O'Regan,

School of Mathematics, Statistics and Applied Mathematics

National University of Ireland, Galway

Ireland.

Email: donal.oregan@nuigalway.ie 